(C) [2009] IEEE. Reprinted, with permission, from Li, Ming., Hoang, Doan., and Li, Zheng 2009, 'Applying Fair Intelligent Congestion Control in a Hybrid QoS Architecture for Wireless Environment', 7th International Conference on Information, Communications and Signal Processing ICICS 2009, pp. 1-6. This material is posted here with permission of the IEEE. Such permission of the IEEE does not in any way imply IEEE endorsement of any of the University of Technology, Sydney's products or services. Internal or personal use of this material is permitted. However, permission to reprint/republish this material for advertising or promotional purposes or for creating new collective works for resale or redistribution must be obtained from the IEEE by writing to pubs-permissions@ieee.org. By choosing to view this document, you agree to all provisions of the copyright laws protecting it. 


\section{Applying Fair Intelligent Congestion Control in a Hybrid QoS Architecture for Wireless Environment}

\author{
Li Zheng and Doan B. Hoang \\ iNEXT - UTS Centre for Innovation in IT Services and \\ Applications, University of Technology \\ Sydney, NSW 2007, Australia
}

\author{
Ming Li \\ School of Engineering and Information Technology \\ Deakin University \\ Geelong, VIC 3217, Australia
}

\begin{abstract}
Differentiated Services (DiffServ) QoS control model is acceptable for the core of the network. However, more explicit and stringent admission and reservation based QoS mechanisms are required in the wireless access segment of the network, where available resources are severely limited and the degree of traffic aggregation is not significant, thus rendering the DiffServ principles less effective. In this paper we present a suitable hybrid QoS architecture framework to address the problem. At the wireless access end, the local QoS mechanism is designed in the context of IEEE 802.11 WLAN with 802.11e QoS extensions. At the edge and over the DiffServ domain, the Fair Intelligent Congestion Control (FICC) algorithm is applied to provide fairness among traffic aggregates and control congestion at the bottleneck interface between the wireless link and the network core.
\end{abstract}

Keywords - hybrid QoS; WLAN; Fair Intelligent Congestion Control; wireless bottleneck.

\section{INTRODUCTION}

From the deployment experiences of Third Generation (3G) mobile networks and Wireless Local Area Networks (WLANs), radio access network is commonly the bottleneck in the end-to-end data path. The QoS limitations of the wireless segment originate from the inherent properties of mobile radio environment $[1,2,3]$. Even though the total resources available over the air interface are, on average, sufficient to meet the total resource requirements of the user application sessions admitted to the system, the level of QoS desired/expected by users may not be provided. Often the system performance for services that are tolerant of longer delay and higher rates of data loss is sacrificed in order to meet the quality of service specified for less tolerant services, on an end-to-end basis.

Therefore, more subtle and explicit QoS control mechanisms are required at the radio access level. QoS mechanisms over the wireless segment of the network have to be designed with consideration given to the entire (end-to-end) network QoS. The drawbacks of QoS architectures proposed thus far vary from insufficient level of control implemented with Differentiated Service (DiffServ) model only, especially over the resource-limited air interface, to scalability and complexity problems of solutions involving end-to-end use of RSVP/IntServ model [3, 4]. In this paper, we present a hybrid QoS architecture framework suitable for new generation wireless IP networks. The proposed hybrid architecture follows the principles of DiffServ model over the core part of the network, and the principles of Integrated Services (IntServ) model locally over the wireless access segment.

With the Hybrid framework, the Fair Intelligent Congestion Control FICC [5, 6, 7] is deployed as an effective rate-based congestion control scheme that addresses both fair bandwidth sharing among traffic classes and congestion problems encountered in current QoS architecture. FICC intelligently predicts per-queue fair share for all traffic aggregates. FICC uses feedback control to keep the Resources Manager operating at a desirable operating point at all times. Resources Manager performs admission control in new flow establishment based on the current usages of the network and nature/class of new flow. FICC also allows overselling bandwidth when the network is not congested to make efficient use of the network resources. At the interface between wired and wireless networks, FICC enables effective admission controls.

\section{HYBIRD QOS ARCHITECTURE}

In general, the network segments would have high volume of available resources and high aggregation of traffic (i.e. core/transport network), and the DiffServ model is useful in providing efficient and scalable QoS control within them. However, because if its aggregation nature, DiffServ mechanisms are not strict enough for controlling QoS where the resources are strongly limited and the levels of traffic aggregation are low, such as in the wireless access network. The last hop (wireless access) radio resource management cannot rely solely on mechanisms providing differentiated treatment of packets that belong to different application sessions. To avoid degradation of QoS as the traffic generated by the users within the same access network increases, a mechanism is needed at the access network level to control the total resource requirements of the sessions admitted to the system (explicit admission control), and to guarantee resources required by each session. IntServ QoS model provides such a mechanism, operating on a session-by-session basis.

For these reasons, we propose a hybrid model whereby FICC-DiffServ principles applied over the core/transport network domain, and IntServ principles applied locally to the QoS control over the wireless access segment. The proposed 
QoS control architecture, as shown in Figure 1, comprises of the Differentiated Services part in the core/transport network segments, and explicit resource management (admission control and reservation) part in the radio access network. Our architecture does not presume any specific QoS control model in the remote network where correspondent node (the other party in the application session) is located; it assumes that it is the other network's responsibility to guarantee at its end a QoS level consistent with that in the remaining parts of the path. The explicit resource management is localized to a single radio access network domain, where stateful and finegranularity control mechanisms operating at the level of individual flows and application sessions can be applied without causing scalability and complexity concerns.

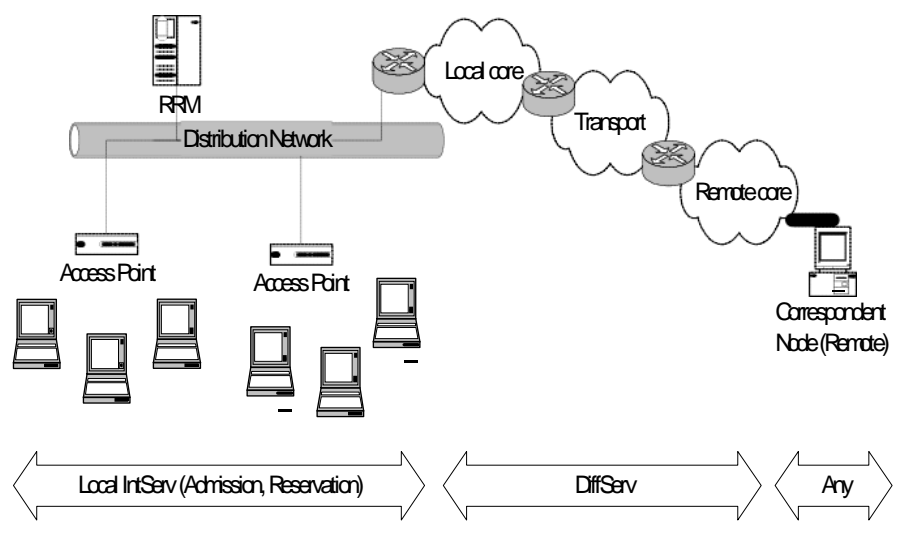

Figure 1 Hybrid QoS Architecture

At the access network level, the resource management is based on functional blocks typical of IntServ model, which is typically equipped with packet (frame) classifier, multiple queues, signalling and service disciplines/policies. They are combined and used to enforce QoS guarantees given to the flows (sessions) upon admission. However, unlike the IETF IntServ architecture, our proposed does not use explicit endto-end path establishment and resource reservations such as those available with RSVP. Particularly, the local level signalling required by the reservation based mechanism does not have to be implemented by means of explicit application level signalling protocol like RSVP, because of being limited in its scope to the last hop. It can be easily implemented at the medium access control level, as part of MAC requests. In case of 3G access networks, the MAC-level admission control and reservation signalling becomes a part of radio resource management necessary to handle admissions of individual terminals and changes of their link states. In case of 802.11 WLANs, the explicit resource management cannot be easily and reliably achieved with the standard MAC data and control frames and random access based Distributed Coordination Function commonly implemented in the current 802.11 products. With supports of QoS extensions to the 802.11 MAC layer, we build MAC level signalling involving exchange of control packets between the wireless station, access point and WLAN-wide radio resource management entities. To enable the exchange of QoS signalling at the local radio access network level, all major entities in the network (wireless stations, access points, edge router) are equipped with QoS agents. The role of QoS agents (management plane processes) is to capture QoS requirements known to the application agents and/or application session control processes, and facilitate the transfer of the QoS related information to QoS processors (classifiers, schedulers) and the exchange of QoS signalling with other QoS (Resource Management) entities.

Central to the admission control, the FICC, an aggregate intelligent congestion control $[5,6,7]$ scheme is adopted particularly at the edge devices to ensure that the domain is not congested to the point that it cannot maintain the agreed level of QoS. FICC plays a key role in Admission Control by suggesting an optimal amount of traffic that should be admitted to maintain an agreeable QoS level. The main purpose of FICC is to achieve the fair bandwidth allocation, minimum buffer queue length variation and simple implementation. It provides the required per-flow QoS response. The flow-level admission control focuses on keeping fairness between individual flows locally to ensure that the available resources are shared fairly with the pre-assigned allocation, while also trying to ensure delay performance when possible.

\section{ADMISSION CONTROL MECHANISM FOR 802.11 WIRELESS LANS}

The IEEE 802.11 standard specifies two modes of operation: Distributed Coordination Function (DCF) and Point Coordination Function (PCF). The 802.11e has been introduced in IEEE to address several QoS limitations of 802.11. The major enhancement in 802.11e is the Traffic Specification (TSPEC) facility that enables flow-based traffic admission and reservation capabilities. When a client wants to reserve resources for a stream of frames (a flow), it sends a TSPEC (Action) frame with specification of the QoS requirements (e.g. data rate, delay) for the flow. The admitted TSPEC is given one of 8 TSPEC IDs (TSIDs) identifying the flow associated with the client in question and all frames using that TSID are classified for treatment according to the TSPEC registered for this specific flow. The TSPEC facility offers a means for MAC level admission control and reservation signalling between the wireless clients

As discussed above, IntServ principles of admission control and reservation are followed locally in the radio access network. Applications, particularly Session-based, normally start with session set-up procedure. The QoS parameters need to be guaranteed for the duration of the session and are either negotiated between the application and network entities at the session set-up time, or implicit in the type of application. Examples include Voice over IP and video streaming sessions. Non-session-based traffic does not need hard QoS guarantees, thus explicit resource reservation is not necessary. The traditional "best effort" service is sufficient for this class of traffic. 
Located at the Radio Resource Manager (RRM), the Admission Control functionality is responsible for admission control of session based application streams. The admission decisions are made on the basis of stream QoS requirements and the current RRM's knowledge of the resource usage (reservation) status in the WLAN. The admitted streams are then registered with the edge router for the purpose of mapping between the 802.11e stream QoS descriptors (TSPEC) and stream identifiers (TSID), the user priority levels on the Ethernet distribution network, and the DiffServ DSCPS visible at the edge of core/transport network. The QoS signalling between the wireless station and the Access Point is accomplished by means of MAC level TSPEC negotiation defined in 802.11e. The signalling between the Access Point, Radio Resource Manager and the edge router is accomplished at the application level (via exchange of IP packets). The Service Differentiation performed at the 802.11e MAC layer level ensures that the high priority (session streams) frames have transmission opportunities (TXOP) satisfying their QoS requirements, as promised at the time of stream admission. Lower priority traffic is treated according to "best effort" principles, filling in the bandwidth available after the sessionbased streams admitted to the system have been satisfied. Figure 2 explains the details of the QoS procedure described.

When a wireless station (STA) initiates, or is invited to, a session-based application, a session set-up dialog is carried out (we may think of a SIP Invite dialog as an example). The QoS agent in the STA will capture the QoS requirements of media streams involved in the session, and map them to a MAC layer TSPEC description as defined in the 802.11e. In order to request admission and reserve radio resources for the stream, an 802.11e ADDTS-Request (add stream request) frame is sent to the AP. It carries a TSPEC element, which describes the source address (MAC), destination address, TSID, and QoS parameters of the stream. The QoS agent in the Access Point then forwards, in an IP packet, the admission request to the Admission Controller in the Radio Resource Manager. The RRM has "global" knowledge of the WLAN resources and reservation status; it will either admit or reject the stream, taking into account the resource usage across the WLAN. If the stream is successfully admitted, the RRM registers the stream with the edge router (via IP level communication) and sends a positive reply to the AP's QoS manager. Subsequently, a QoS ADDTS-Response frame is sent back to the wireless station, carrying a TSPEC element for the admitted stream. The admitted TSPEC could be as requested, or altered as a result of resource negotiation at the RRM.

Once resources are reserved for a stream, application data frames must be classified in order for the service differentiation mechanism to be applied in the AP at the MAC layer level. The task of the classifier (for downlink traffic, in the edge router) is as follows: given an IP datagram of a particular flow (identified by, for example, the source/destination IP addresses and port numbers; we will refer to this as the flow ID), allocate the stream identifier (TSID) to the corresponding MAC data frame. For user data traffic on the uplink, the classification is straightforward because the QoS agent in the wireless station has knowledge of the streams generated by this station and admitted to the system. As a result, the TSID can be inserted directly into the MAC frame when it is generated at the station. For downlink traffic, classification is more complex because the AP operates only at the MAC layer level, and has no knowledge of the traffic flows at the IP level. The process of classification and mapping of IP flows onto the TSIDs must begin at a layer 3 device, i.e. the edge router, as below.

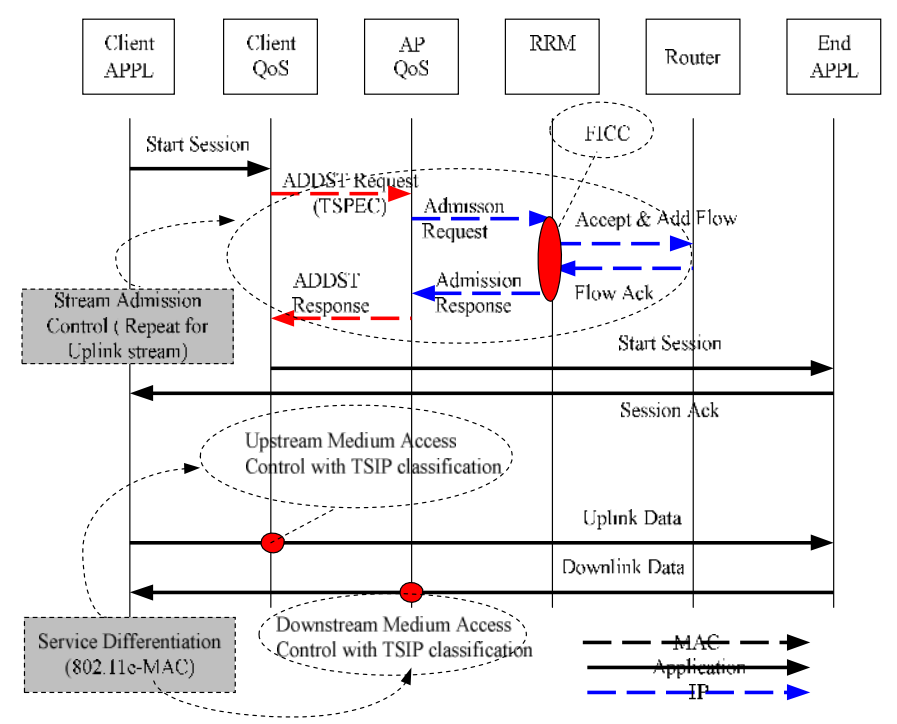

Figure 2 A Signalling Diagram for Flow Admission Procedure

In details, that, for downlink traffic, the edge router examines IP packets to detect flows and marks the distribution network (e.g. Ethernet) MAC frames with a priority level based on the TSID previously registered for the flow (recall that as part of admission control procedures, the RRM notifies the edge router of new flows). The priority information in the 802.3 MAC frame on the distribution network is carried in the additional 802.1p header (this additional header, which can be processed by most Ethernet products available today, carries a 3-bit user priority field). Therefore, when the router sends a distribution network MAC frame towards the AP, the frame contains the mobile host MAC address and the user priority value equal to the registered TSID. The AP's QoS agent must then interpret the Ethernet user priority field as the TSID for this frame. Together with the identity of the destination station, this determines the service differentiation treatment the frame will receive at the AP.

In support of classifying downlink traffic using the Ethernet user priority field, the network should be configured as below. The WLAN distribution network should be separated from other parts of the LAN by an edge router where classification of flows is performed. Fixed hosts attached to the Ethernet (such as servers within the WLAN subnet) must be equipped with a QoS agent that ensures MAC 
frames sent by them are marked with the appropriate user priority that will be interpreted by the APs as TSID. These seem to be practical to most of recent Ethernet subnet today.

\section{FAIR InTELligenge CONGESTION CONTROL As ThE CORE OF ADMISSION CONTROL}

The above mechanism is built to provide an effective way to convey traffic information included in the TSPEC, between QoS Agent in the client/applications and RRM/AP. Based on such information, RRM make admission decisions and inform the QoS Agent with the same channel. Performances on the admission control rely on such decision and its algorithms below. Here, we examine the effectiveness and efficiency of FICC, when applying to our hybrid model.

The purpose of such admission control functions is to prevent congestion at the edge as well as within a DiffServ domain and to allocate resources fairly among traffic classes within the domain. It uses available resource information updated to calculate an Explicit Rate (ER) for each class. Perflow admission control guarantees the fairness among individual flows within the same class. By doing, the control algorithm firstly attempts to maintain the queue length at the bottlenecked router along the path of the session close to a target point to avoid router buffer overflow and underflow. The bottlenecked router always operates at the full capacity of the output link without interruption from traffic congestion or buffer starvation. Thus, the most efficient throughput can be achieved. In addition, variations on queue length and consequently queuing delays are reduced. On the other hand, FICC attempts to allocate the available bandwidth fairly. Specifically, FICC tries to allocate bandwidth equally among aggregates (DSCPs) with equal status and to distribute the unused bandwidth (left over by constrained aggregates) fairly among the aggregates that can use an additional share. To achieve this objective, FICC oversells bandwidth when the network operates below the target point. And each sender is continuously informed about its current fair share based on the dynamic network traffic conditions by the feedback message.

$$
\begin{array}{ll}
f(Q)=\frac{\text { Buffer_Size }-Q_{-}}{\text {Buffer_Size }-Q_{0}} & \text { for } Q>Q_{0} \\
f(Q)=\frac{(a-1)^{*}\left(Q_{0}-Q\right)}{Q_{0}}+1 & \text { for } Q \leq Q_{0}
\end{array}
$$

To achieve such, it is thus essential to relate appropriately the buffer queue length to the degree of network congestion. We use Mean Allowed Class Rate (MACR) to measure the estimated fair share of the aggregate. This MACR in turn is based on the queue length at the router and determines the explicit rate (ER) of an aggregate (the maximum rate at which the network informs the source of the aggregate that it can support). The "queue control function" is expressed using Buffer Utilization Ratio (BUR) of an output queue as the target percentage of buffer capacity that should be occupied. When the target is met, the queue occupancy is Buffer_Size
*BUR. This target occupancy is designed to avoid link underutilization and the remaining buffer capacity Buffer_Size* $(1-B U R)$ is available to absorb packets that might arrive in the queue when the network becomes highly loaded. While BUR defines the target buffer operating point, the corresponding target queue length $Q O(=B U R *$ Buffer_Size) is often referred to instead of BUR. Since the queue builds up and drains out continuously, the congestion function should be continuous to regulate smoothly the queue fluctuations through the computed ER values. A sophisticated and simple queue control function, the piecewise linear congestion function $f(Q)$ is shown in (1). It would fine-tune the performance of the congestion control algorithm, however, it should also be pointed out that BUR only indicates the desirable long-term operational level. The actual buffer utilization fluctuates around this level.

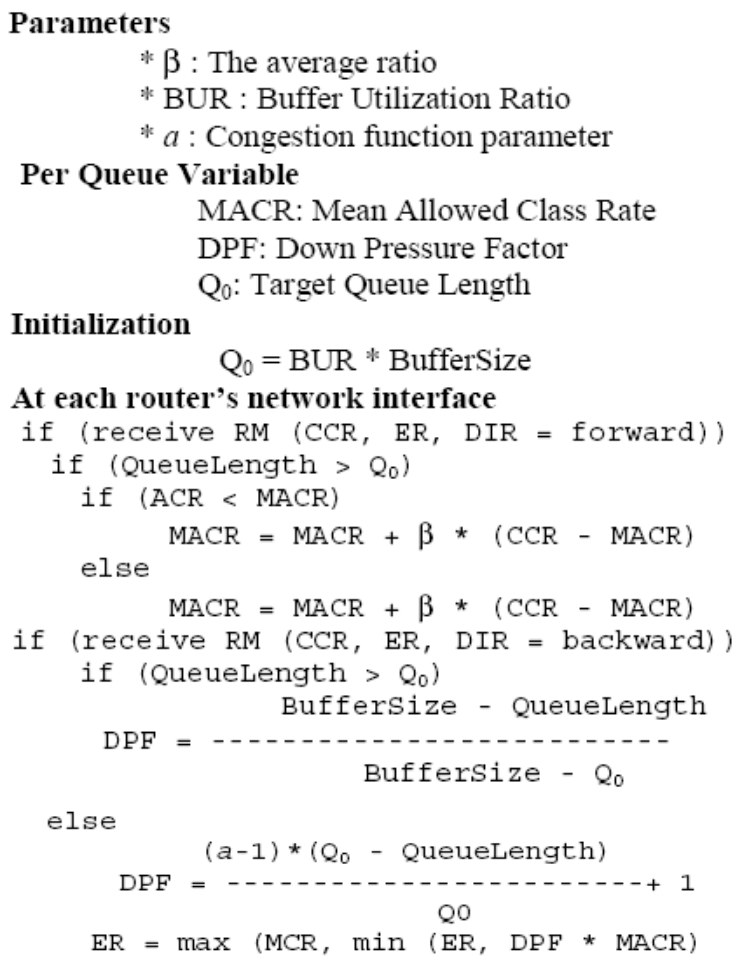

The actual algorithm is described here. The target is to estimate its available bandwidth and advise the traffic sources appropriately. Firstly, the current traffic rate of all aggregates passing through it are estimated and allocated the available bandwidth fairly among its aggregates. As below, the MACR contained in TSPEC is updated with the exponential average factor, which is a true exponential running average of the current load from all aggregates only when the network operates below the target operating point. When the network exceeds the target operating point, FICC does not allow MACR to increase further. That means that MACR does not track any ACR value larger than the current MACR when the queue is congested. This rule prevents all those aggregates whose ACRs are already equal larger than the current MACR to increase their rates further, thereby preventing further loading of the network. Instead, all aggregates have to reduce 
their rates to the same explicit rate and the throttling is performed fairly. However, when the network operates below the target operating point, all aggregates are allowed to increase their rate by a factor greater than 1 (that is what we mean by overselling), which enables aggregates that are capable of using the available bandwidth to take advantage of it. The explicit rate is calculated as above.

The Resource Discovery (RD) protocol is responsible of ensuring communications FICC in RRM and QoS Agent in clients. Its agent in AP monitors available resources of wireless links (in MAC level) and provide such feedback to FICC. Such feedback includes MACR and ER. With the supports of the signalling mechanism we discussed in Section 3 , RD information captures the resources availability and is conveyed in a TSPEC frame among RRM/AP and client using the mechanism we proposed. In RRM, information is generated, calculated and updated, then is sent to AP for execution of the determination to support clients' demand. With supports of RD and signalling mechanism discussed above, FICC manage the resources among traffic flows based on the algorithms and mechanism above.

\section{SimULATION ANALYSIS}

Network simulator ns2 was used to evaluate the framework, where both last hops of DiffServ Domain are wireless LAN. Several agents were designed in $\mathrm{C}++$ to implement the schemes. The simulation topology is shown in Figure 1, where FICC is implemented particularly in AP and RRM, where wireless link is the bottleneck of network at 1 Mpbs bandwidth avaliable. The bandwidths and propagation delays of wired links are standard. There would be four classes of traffic, AF11 (Gold), AF21 (silver), AF31 (Bronze), and Best Effort, who claim 40\%, 30\%, 20\%, and 10\% respectively. They are mixture of TCP and UDP traffic in which Best Effort has UDP traffic and other three classes only have TCP traffic (in Gold, Silver, and Bronze). In the simulation, UDP traffic has constant bit rate is $1 \mathrm{Mbps}$ so that it causes the bottleneck. The simulation results for FICC and regular DiffServ permitted performance in terms of queue length, packet loss, end-to-end delay, throughput, goodput and fairness. The throughput is defined as the number of bits of all the TCP packets transmitted at the source (including RD packets if FICC was used) divided by the duration of the transmission. The goodput is defined as the number of bits of TCP packets transmitted at the source and successfully received at the destination divided by the duration of the transmission.

\section{A. Violation Prevention and Congestion Control}

We firstly look at how the FICC could prevent traffic violation from certain classes, in order to avoid traffic congestion. In Figure 3, with regular DiffServ schemes, silver class traffic does occupy most of bandwidth, exceeding the gold class indeed, as it has shorter RTT, 32ms. Obviously, sliver class violates the traffic condition regarding of the class it has been assigned (sliver). The damage is that gold class and others could not be allocated for bandwidth fairly, as it has been agreed. Furthermore, the gold class actually experiences lower throughput at $0.28 \mathrm{Mpbs}$ in average, as shown in Figure 3 and longer delay with larger queue length.

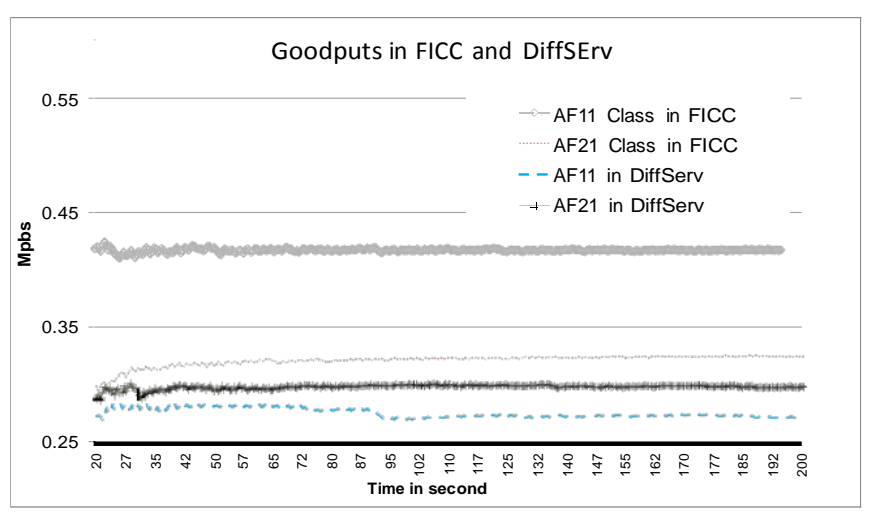

Figure 3 Goodput comparisons between FICC and DiffServ

In FICC, the queue length around the target point is controlled; there is no congestion and no packet loss due to congestion during the transmission. The queue length variation and average queue length with FICC are also smaller than those under regular DiffServ. In Figure 4, this has been proven in the gold class traffic, as its queue length has been largely improved from average 60 to 20 as shown. In fact, under regular DiffServ scheme, there is sharp fall after the queue reaches 80, due to the queue length limitation and packet timeout. However, this does not happen under our FICC scheme.

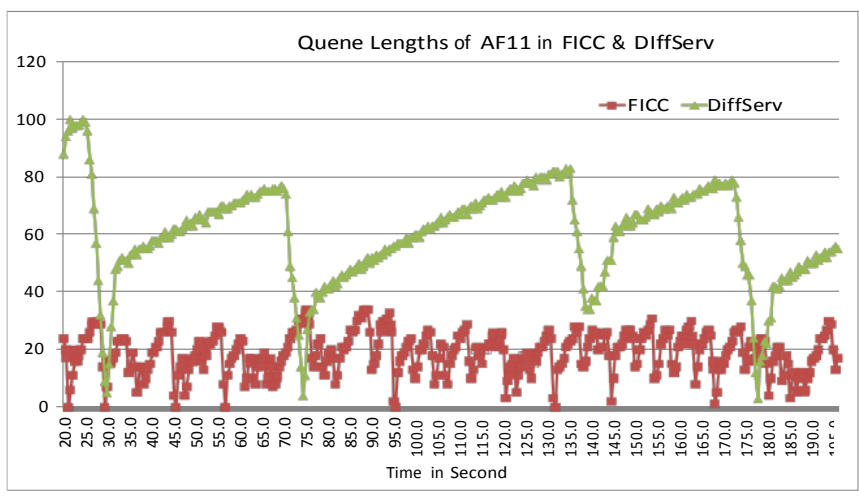

Figure 4 Queue Performance Comparisons between FICC and DiffServ

\section{B. Fair Allocation of Resources}

As above, DiffServ could not prevent any traffic violation. In this section, we investigate how FICC fairly allocate resources to different classes/PHB. As discussed, FICC always accurately estimates the fair share for each session at each router and constantly conveys the information to sender by RD and ACK packets. Based on these feedbacks, traffics are policed and sessions with FlCC should share roughly the assigned amount of bandwidth. In Figure 5 below, it shows all four classes are allocated with 10\%, 20\%, 30\% and 40\% bandwidth, as agreed in the Admission Control. Particularly, in BE class, the UDP is carried with a constant bit rate of 1 Mpbs, which would overload the system, particularly in the 
wireless section. However, FICC could minimise its impact and fairly distribute the resources. In Figure 6, the queue length comparison between $\mathrm{AF} 11$ and $\mathrm{BE}$ shows us such fair distribution, as AF11 queue constantly maintain at average of 20 even it is already allocated $40 \%$ bandwidth, while BE may suffer itself as its large request for resources while only $10 \%$ is allocated.

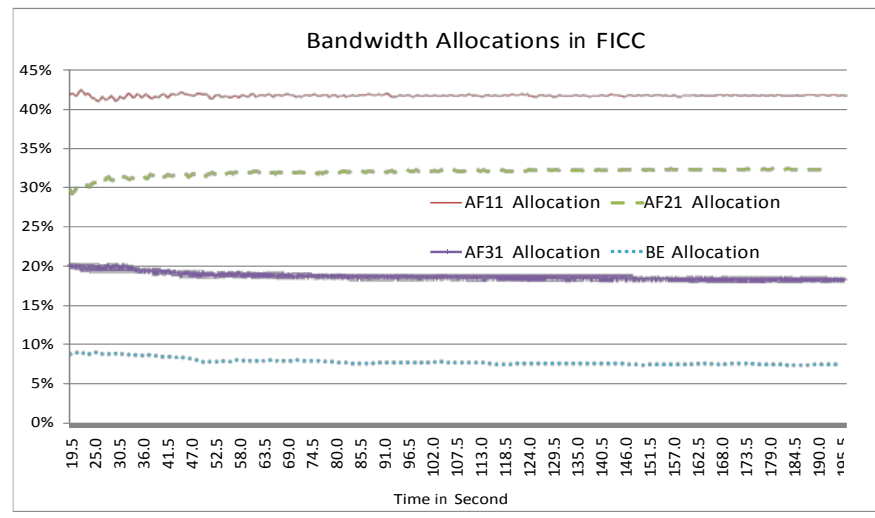

Figure 5 Resources Allocation of Bandwidth in FICC

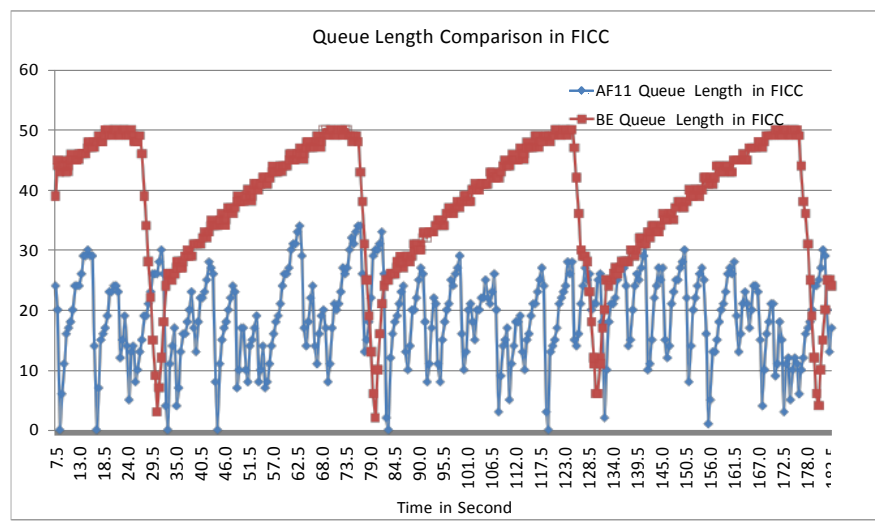

Figure 6 Resources Allocation of Queue Length in FICC

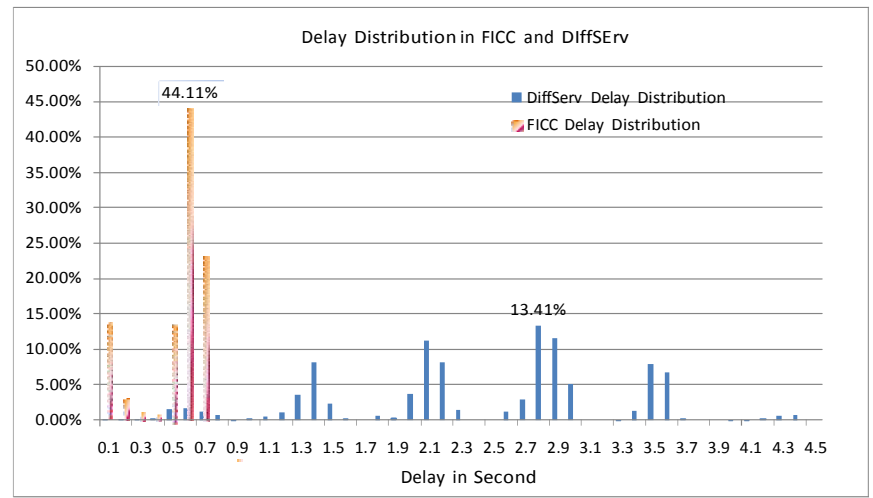

Figure 7 Delay Distributions in FICC and DiffServ

\section{Delay Performance}

We also provide the end-to-end delays performance comparison for regular DiffServ and FICC schemes. As the FICC not only could prevent traffic violation, but also provide intelligent admission control, which ensure the delay performance as well. In Figure 7, the majorities of delays in FICC are within 0.5 second to 0.7 second, while the delay performance in DiffServ ranges mainly from 1.0 second to 3.7 second. Furthermore, the delay distribution analysis below shows that in FICC, $44.11 \%$ contribution comes from 0.6 second, while delays in regular DiffServ spread across 0.5 and 3.7 with a maximum of $13.41 \%$ at 2.8 second delay. This indicate the jitter, delay variation in regular DiffServ is much worst than FICC. That could be a great impact in the delaysensitive applications, such as Voice over IP.

\section{CONCLUSION}

The paper has presented a hybrid QoS architecture framework for next generation wireless networks. The framework deploys an FICC-DiffServ-enhanced QoS control model for the core/transport part of the end-to-end path, but applies IntServ principles of explicit admission control and resource reservation locally only in the wireless access network domain. In the context of IEEE 802.11 WLAN with 802.11e QoS extensions, a flow signaling mechanism has been designed to meet the proposed explicit admission control and resource reservation locally in the wireless access network. Particularly, within the admission control, FICC is adopted as its algorithm at the interface of the hybrid framework. Simulation results have shown that FICC manages effectively the overloading scenario in the edge section, which is the resources bottleneck of the wireless access domain. Particularly, it prevents traffic violation from uncontrolled UDP traffic, provides guarantee to those priority traffic in terms of guaranteed bandwidth allocation and specified delay. The results demonstrate that such a proposed hybrid framework with the Fair Intelligent Congestion Control can be realized for effective end-to-end QoS delivery. Further study should address the parametric analysis for FICC.

\section{REFERENCES}

[1] Maniatis, S.; Grecas, C.; Venieris, I., "End-to-end quality of service issues over next generation mobile Internet", Communications and Vehicular Technology, 2000. Symposium on, P150-154

[2] Dixit, S.; Yile Guo; Antoniou, Z., "Resource management and quality of service in third generation wireless networks", IEEE Communications Magazine, Volume: 39 - 2, Feb. 2001; P125 -133

[3] Koodli, R.; Puuskari, M., "Supporting packet-data QoS in next generation cellular networks", IEEE Communications Magazine, Volume: 39; Issue: 2, Feb. 2001; P180-188

[4] García-Macías, I. A., Rousseau, F., Berger-Sabbatel, G Toumi, L, Duda, A "Quality of service and mobility for the wireless internet", Proceedings of the first workshop on Wireless mobile internet July 2001, P34 - 42 .

[5] Hoang, D.B., Phan, H. T, "End-Diff: An End-to-End QoS Architecture," International Symposium on Communications and Information Technologies (ISCIT 2007), Sydney, October 2007.

[6] Phan, H. T., Hoang, D. B, "FICC-DiffServ: A New QoS Architecture Supporting Resources Discovery, Admission and Congestion Controls," International Conference on Information Technology and Applications (ICITA), 2005.

[7] H. T. Phan, Hoang, D. B, "Extension of BGP to support multi-domain FICC-Diffserv architecture," presented at IEEE Conference on Advanced Information Networking and Applications (AINA06), Vienna. 